\title{
Interface slip between two half-planes caused by a steadily moving force
}

\author{
R.D. LOW * and MARIA COMNINOU \\ Mechanical Engineering and Applied Mechanics, University of Michigan, Ann Arbor, MI 48109, USA
}

(Received August 18, 1983)

\begin{abstract}
A computationally simple problem is devised to study the behavior of steadily moving slip zones. Results are presented graphically.
\end{abstract}

\section{Introduction}

Frictional interface slip due to moving loads has been the subject of renewed interest $[1,2]$ following the earlier work of Anscombe and Johnson [3]. One of the interesting differences between moving and stationary loads is the absence of slope discontinuity or "hook" in the shear tractions at the trailing edge of the moving slip zone. The problem considered in [1,2] involved forces moving steadily over the surface of a layer which was pressed against a half plane of identical elastic constants. Because this problem is computationally complex, a simpler problem is considered here to bring out the salient features of moving slip zones. The layer is replaced by another half plane and the surface force by an internal force. Then the solution can be obtained in closed form but is of great algebraic complexity. Only the case of a force parallel to the interface is examined. In addition to applied pressure, the solids are also sheared at infinity.

\section{Formulation and solution}

Two half planes with similar elastic constants are pressed and sheared at infinity with applied tractions $p^{\infty}, q^{\infty}$ respectively. A force $P$ moves parallel to the interface at distance $h$ with velocity $c$ to the right as shown in Fig. 1. We assume that the motion is quasistatic and neglect inertia effects. The fixed coordinates are denoted by $\hat{x}, \hat{y}$ and the coordinates moving with $P$ by $x, y$ so that

$$
x=\hat{x}-c t, \quad y=\hat{y} .
$$

\footnotetext{
* Deceased.
} 
As long as the interface adheres, the interface tractions are given by the Kelvin solution [4]:

$$
\begin{aligned}
& \sigma_{y y}(x, 0)=-p^{\infty}-\frac{(\kappa-1) P}{2 \pi(\kappa+1)} x \frac{\frac{5-\kappa}{\kappa-1} h^{2}-x^{2}}{\left(x^{2}+h^{2}\right)^{2}}, \\
& \sigma_{x y}(x, 0)=q^{\infty}+\frac{(\kappa+3) P}{2 \pi(\kappa+1)} h \frac{\frac{\kappa-1}{\kappa+3} h^{2}+x^{2}}{\left(x^{2}+h^{2}\right)^{2}},
\end{aligned}
$$

where $\kappa=3-4 \nu$ for plane strain.

Stick prevails as long as

$$
-\boldsymbol{\sigma}_{y y}(x, 0) f>\left|\sigma_{x y}(x, 0)\right|, \quad \text { all } x,
$$

where $f$ is the coefficient of friction.

By (2), (3), and (4) it is determined that slip starts at a point $\xi=x / h$ corresponding to the real root of

$$
(\kappa-1) \xi^{4}+\frac{2(\kappa+3)}{f} \xi^{3}-12 \xi^{2}-\frac{2(5-\kappa)}{f} \xi+5-\kappa=0,
$$

which gives the smallest $P$. This root occurs in the range

$$
-\left(\frac{5-\kappa}{\kappa+3}\right)^{1 / 2}<\xi<-\left(\frac{5-\kappa}{6+\left(41-6 \kappa+\kappa^{2}\right)^{1 / 2}}\right)^{1 / 2},
$$

and at a load

$$
P^{*}=\frac{P / h p^{\infty}}{f-q^{\infty} / p^{\infty}}=\frac{2 \pi(\kappa+1)\left(\xi^{2}+1\right)^{2}}{(\kappa-1) f \xi^{3}+(\kappa+3) \xi^{2}-(5-\kappa) f \xi+\kappa-1} .
$$

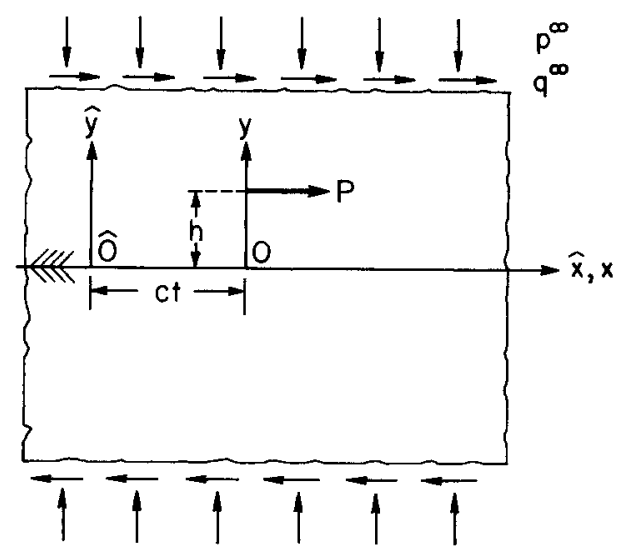

Figure 1. Geometry of the problem. 
Separation begins at

$$
\xi=-\left(\frac{5-\kappa}{6+\left(41-6 \kappa+\kappa^{2}\right)^{1 / 2}}\right)^{1 / 2}
$$

when the load reaches the value

$$
P^{* *}=\frac{P}{h p^{\infty}}=\frac{64 \pi(\kappa+1)\left[\kappa+1+\left(41-6 \kappa+\kappa^{2}\right)^{1 / 2}\right]\left[6+\left(41-6 \kappa+\kappa^{2}\right)^{1 / 2}\right]^{1 / 2}}{(5-\kappa)^{3 / 2}\left[\kappa+5+\left(41-6 \kappa+\kappa^{2}\right)^{1 / 2}\right]^{2}}
$$

These expressions are plotted in Fig. 2 for varying $\kappa$ and $f=0.5$. Figure 3 shows the effect of friction on the initiation of slip. Note that the results are valid for

$$
f>q^{\infty} / p^{\infty},
$$

outside which gross slip occurs.

We anticipate that slip occurs before separation and restrict attention to the case of slip only. It is expected that a single slip zone appears first along the negative $x$-axis in the interval $-a<x<-b$. We represent the slip zone by a distribution of glide dislocations with density $B(\xi)$. The tractions due to $B(\xi)$ are then [5]

$$
\begin{aligned}
& \tau_{x y}(x, 0)=\frac{2 \mu}{\pi(1+\kappa)} \int_{-a}^{-b} \frac{B(\xi)}{x-\xi} \mathrm{d} \xi, \\
& \tau_{y y}(x, 0)=0 .
\end{aligned}
$$

Hence the total tractions along the interface are

$$
N(x)=\sigma_{y y}(x, 0),
$$

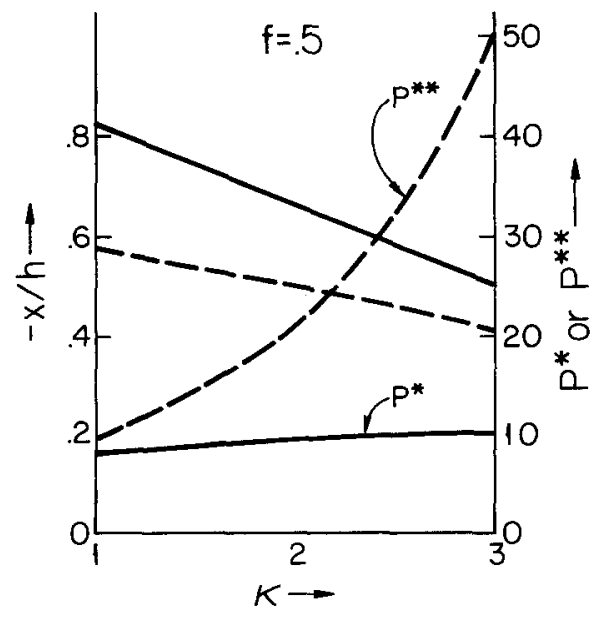

Figure 2. Solid lines: location of initiation of slip and respective dimensionless load $P^{*}$. Dashed lines: location of initiation of separation and respective dimensionless load $P^{* *}$.

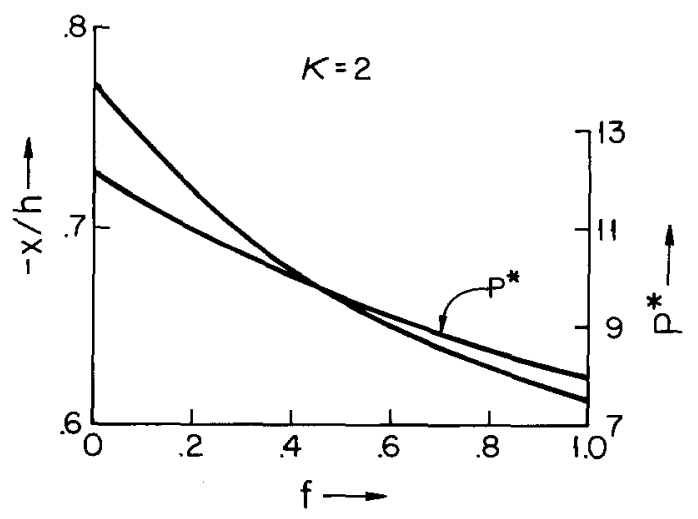

Figure 3. Location of initiation of slip and respective dimensionless load $P^{*}$ versus friction. 


$$
S(x)=\frac{2 \mu}{\pi(1+\kappa)} \int_{-a}^{-b} \frac{B(\xi) \mathrm{d} \xi}{x-\xi}+\sigma_{x y}(x, 0),
$$

where $\sigma_{y y}(x, 0)$ and $\sigma_{x y}(x, 0)$ are given by (2) and (3). Note that $B(x)$ does not affect the normal tractions, and therefore (8) and (9) defining the onset of separation remain valid although slip occurs first.

The boundary conditions that must be satisfied in the slip zone are

$$
\begin{aligned}
& |S(x)|=f|N(x)| \quad-a<x<-b, \\
& \operatorname{sgn} S(x)=\operatorname{sgn} B(x),
\end{aligned}
$$

according to Coulomb's law of friction.

In addition

$$
N(x) \leqslant 0, \quad-\infty<x<\infty,
$$

which implies that the load $P / h p^{\infty}$ must be restricted by the value given in (9) so that no separation occurs. Outside the slip zone the shear tractions must not exceed the maximum static friction

$$
|S(x)|<-f N(x)
$$

Anticipating positive slip, we can write (15) as

$$
\begin{aligned}
& \frac{2 \mu}{\pi(\kappa+1)} \int_{-a}^{-b} \frac{B(\xi) \mathrm{d} \xi}{x-\xi}+q^{\infty}+\frac{(\kappa+3) P}{2 \pi(\kappa+1)} h \frac{\frac{\kappa-1}{\kappa+3} h^{2}+x^{2}}{\left(x^{2}+h^{2}\right)^{2}} \\
& \quad=f\left[p^{\infty}+\frac{(\kappa-1) P}{2 \pi(\kappa+1)} x \frac{\frac{5-\kappa}{\kappa-1} h^{2}-x^{2}}{\left(x^{2}+h^{2}\right)^{2}}\right], \quad-a<x<-b .
\end{aligned}
$$

The solution of (19) can be obtained in closed form [6]. Since a solution bounded at both ends is required [7], a consistency condition must be satisfied. Moreover, the two conditions (16) and (18) are equivalent to continuity of slope of $S(x)$ at the trailing edge of the slip zone [1] or equivalently

$$
\left.\frac{\mathrm{d} B(x)}{\mathrm{d} x}\right|_{x=-a}=0 \text {. }
$$

The consistency condition and (20) yield two algebraic equations for the determination of $a$ and $b$ :

$$
\begin{aligned}
& \frac{P / h p^{\infty}}{f-q^{\infty} / p^{\infty}}=\frac{2 \sqrt{2 \pi}(\kappa+1)}{F(\alpha, \beta, \kappa, f)}\left[\left(1+\alpha^{2}\right)\left(1+\beta^{2}\right)\right]^{5 / 4} \\
& F(\alpha, \beta, \kappa, f)=\left\{(\alpha+\beta)(1+\alpha \beta)-f\left[(\kappa-1) \alpha^{2} \beta^{2}+(\kappa-2)\left(\alpha^{2}+\beta^{2}\right)\right.\right. \\
&+\kappa-3]\}\left[1+\frac{\alpha \beta-1}{\left[\left(\alpha^{2}+1\right)\left(\beta^{2}+1\right)\right]^{1 / 2}}\right]^{1 / 2} \operatorname{sgn}(\alpha+\beta) \\
&+\left\{(\kappa+1) \alpha^{2} \beta^{2}+\kappa\left(\alpha^{2}+\beta^{2}\right)+(\kappa-1)\right. \\
&+f(\alpha+\beta)(\alpha \beta+1)\}\left[1-\frac{\alpha \beta-1}{\left[\left(\alpha^{2}+1\right)\left(\beta^{2}+1\right)\right]^{1 / 2}}\right]^{1 / 2},
\end{aligned}
$$




$$
\begin{aligned}
& \left\{\left[-(\kappa-2)-(7-\kappa) f \alpha-(\kappa+4) \alpha^{2}+(\kappa-1) f \alpha^{3}\right]\right. \\
& \left.\quad+[1-\alpha \beta-(\alpha+\beta) f] \frac{\alpha^{2}+1}{\beta^{2}+1}\right\}\left[1+\frac{\alpha \beta-1}{\left[\left(\alpha^{2}+1\right)\left(\beta^{2}+1\right)\right]^{1 / 2}}\right]^{1 / 2} \operatorname{sgn}(\alpha+\beta) \\
& \quad+\left\{\left[(4-\kappa) f+(5-\kappa) \alpha-(\kappa+2) f \alpha^{2}-(\kappa+1) \alpha^{3}\right]\right. \\
& \left.\quad+\left[\alpha+\beta+(1-\alpha \beta) f \frac{\alpha^{2}+1}{\beta^{2}+1}\right]\right\}\left[1-\frac{\alpha \beta-1}{\left[\left(\alpha^{2}+1\right)\left(\beta^{2}+1\right)\right]^{1 / 2}}\right]^{1 / 2} \\
& \quad=0
\end{aligned}
$$

where

$$
\alpha=a / h, \quad \beta=b / h .
$$

The values of $\alpha$ and $\beta$ versus the dimensionless loading parameter $P^{*}$ are shown in Fig. 4.

The shear tractions are of the form

$$
S(x)= \begin{cases}-f N(x)-Q(x), & x<-a, \\ -f N(x), & -a<x<-b, \\ -f N(x)+Q(x), & -b<x\end{cases}
$$

where $Q(x)$ is a complicated algebraic function involving $a, b, f$ and $\kappa$. For sufficiently high values of the load, a second slip zone starts along the positive $x$-axis. The location and load at which the second slip zone initiates can be computed by the requirements

$$
Q(x)=0, \quad Q^{\prime}(x)=0 .
$$

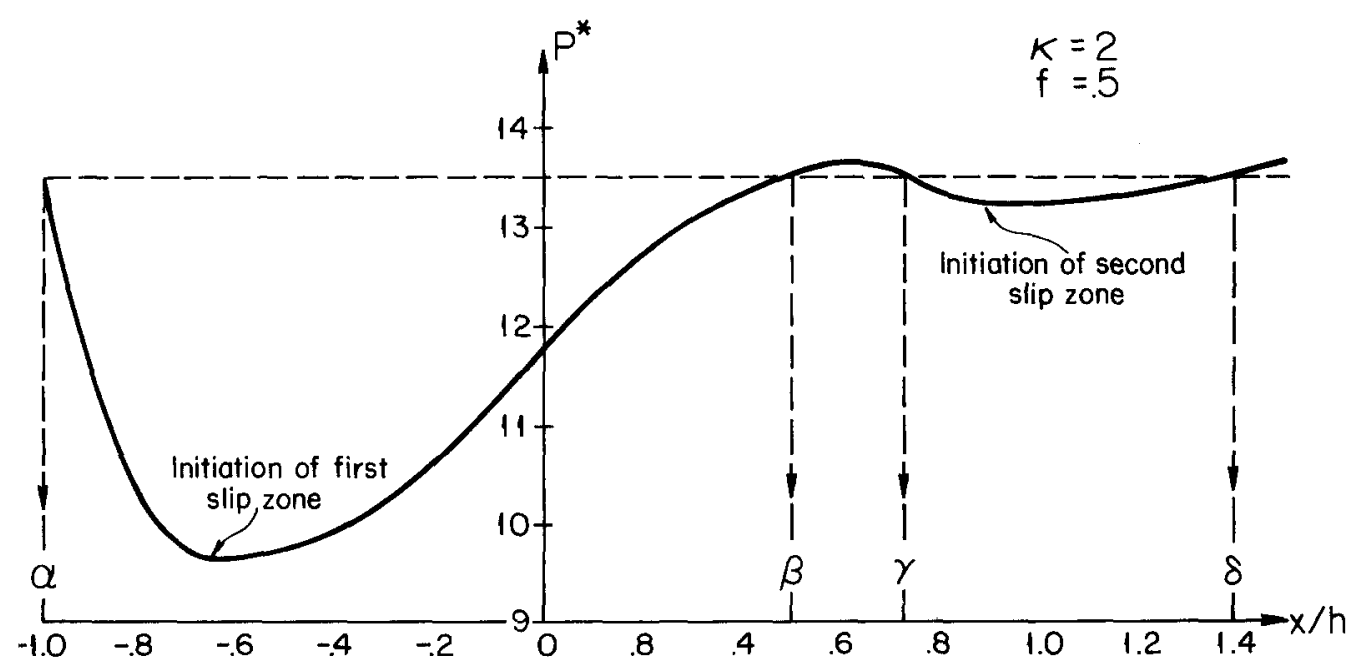

Figure 4. Slip zones end points $\alpha, \beta, \gamma, \delta$ versus dimensionless load $P^{*}$. 
For $\kappa=2$ and $f=0.5$ the second slip zone starts at $x / h-3.8$ and at load

$$
\frac{P / h p^{\infty}}{f-q^{\infty} / p^{\infty}}=13.3 \text {. }
$$

When two slip zones are present, (19) remains valid provided that the integration is extended over both intervals. We now have four unknown parameters, i.e. the end points of the slip zones and four auxiliary conditions, i.e. two consistency equations and two conditions requiring continuity of $B^{\prime}(x)$ at the trailing edges of the slip zones. The extent of the slip zones are shown in Fig. 4, where $\gamma$ and $\delta$ denote the normalized values of the end points of the second slip zone. As the load is increased, the two slip zones coalesce into a single slip zone for which the original formulation remains applicable.

\section{Acknowledgment}

Support by the Office of Naval Research through contract N00014-81-K-0626 is gratefully acknowledged.

\section{References}

[1] M. Comninou, J.R. Barber and J. Dundurs, Interface slip caused by a surface load moving at a constant speed. International Journal of Mechanical Sciences 25 (1983) 41-46.

[2] F.K. Chang, M. Comninou and J.R. Barber, Slip between a layer and a substrate caused by a normal force moving steadily over the surface. International Journal of Mechanical Sciences 50 (1983) 803-809.

[3] H. Anscombe and K.L. Johnson, Slip of a thin solid tyre press-fitted on a wheel. International Journal of Mechanical Sciences 16 (1974) 329-334.

[4] S. Timoshenko and J.N. Goodier, Theory of Elasticity, 3rd edn. McGraw-Hill (1970).

[5] J. Dundurs, Elastic interaction of dislocations with inhomogeneities. In: Mathematical Theory of Dislocations, The American Society of Mechanical Engineers (1970).

[6] N.I. Muskhelishvili, Singular Integral Equations, P. Noordhoff (1953).

[7] J. Dundurs and M. Comninou, Some consequences of the inequality conditions in contact and crack problems. Journal of Elasticity 9 (1979) 71-82. 